\title{
Burden of H1N1 Influenza Related Mortality in a Tertiary Care Centre
}

\author{
Supriya Prathibha Shankaranarayana Bhat ${ }^{1}$, Mahalingeshwara Kelagina Pidamale Bhat ${ }^{2}$, \\ Ruhi Kumar ${ }^{3}$, Giridhar Belur Hosmane ${ }^{4}$, Ivor D'Sa ${ }^{5}$ \\ 1,2,3,4,5 Department of General Medicine, K.S. Hegde Medical Academy, Nitte (Deemed to Be University), \\ Deralakatte, Mangalore, Karnataka, India.
}

\section{ABSTRACT}

\section{BACKGROUND}

Influenza or the common flu is a commonly recognized cause of respiratory tract infection. A high level of clinical suspicion is required before a sample can be sent and often the diagnosis is established posthumously. In developing countries like India, data on disease burden of Influenza is severely lacking. It is a consequence of an under-estimation of Influenza as an important cause of illness and its natural course under-simplified to be a mild self-limiting illness. India was affected with a moderate severity compared to the other countries, although there was an insufficiency of epidemiological data from India during 2009 - 2010. Post-pandemic period, India was affected by major outbreaks in 2015 and 2017 . We wanted to analyse the rate of positivity in a tertiary care centre amongst the total number of oropharyngeal swabs sent for Influenza virus testing. We also wanted to assess the mortality rate in Influenza positive cases in a tertiary care centre.

\section{METHODS}

Data of oropharyngeal swabs sent for influenza virus testing through Real-Time Reverse Transcription Polymerase Chain Reaction from K.S. Hegde Medical Academy, Deralakatte was reviewed through a retrospective search of official influenza virus reports of Karnataka State along with the reports in Medical Records Department of K.S. Hegde Medical Academy in the time period of January $1^{\text {st }} 2016$ to November $22^{\text {nd }}$ 2018. This study was done as a cross sectional retrospective study.

\section{RESULTS}

Out of 127 oropharyngeal swabs that were sent during the study time period, 24 (18.9 $\%$ ) were positive for influenza virus. 18 (75 \%) cases were found to be H1N1 positive, 3 were H3N2 positive (seasonal strain) and remaining 3 were positive for Influenza B virus. 9 (37.5\%) out of 24 cases of influenza succumbed to the disease, of which all were $\mathrm{H} 1 \mathrm{~N} 1$ positive ( $50 \%$ of the total $\mathrm{H} 1 \mathrm{~N} 1$ cases).

\section{CONCLUSIONS}

H1N1 related mortality is a cause for concern for every healthcare providing institution. Influenza virus should be actively tested in patients with respiratory symptoms. During this COVID 19 related pandemic situation, there is a higher possibility of underdiagnosing H1N1. Lessons learnt from the past should be remembered and Influenza should not be neglected.

\section{KEY WORDS}

H1N1, Influenza Related Mortality
Corresponding Author: Dr. Ruhi Kumar, Room 102, Kshema Ladies Hostel, Deralakatte - 575108, Mangalore, Karnataka, India.

E-mail: ruhikmbbs@yahoo.com

DOI: $10.14260 / \mathrm{jemds} / 2020 / 858$

How to Cite This Article:

Bhat SPS, Bhat MKP, Kumar R, et al. Burden of H1N1 influenza related mortality in a tertiary care centre. J Evolution Med Dent Sci 2020;9(52):3917-3920, DOI: $10.14260 /$ jemds/2020/858

Submission 04-09-2020, Peer Review 01-11-2020, Acceptance 09-11-2020, Published 28-12-2020.

Copyright (c) 2020 Supriya Prathibha Shankaranarayana Bhat et al. This is an open access article distributed under Creative Commons Attribution License [Attribution 4.0 International (CC BY 4.0)] 


\section{BACKGROUND}

Influenza or the common flu is a commonly recognized cause of respiratory tract infection. A high level of clinical suspicion is required before a sample can be sent and often the diagnosis is established posthumously. In developing countries like India, data on disease burden of influenza is severely lacking. ${ }^{1}$ It is a consequence of an under-estimation of influenza as an important cause of illness and its natural course under simplified to be a mild self-limiting illness. It is slowly becoming an important differential diagnosis to be ruled out, especially in people who deteriorate quickly requiring respiratory support. The 2009 H1N1 pandemic is what brought this disease to the forefront, gaining awareness.

The influenza virus is part of the Orthomyxoviridae family. It has three types: A, B and C. The influenza A virus is subdivided and named based on two cell surface glycoproteins: haemagglutinin $(\mathrm{H})$ and neuraminidase $(\mathrm{N}) .^{2}$ The H1N1 strain responsible for the pandemic in 2009 originated and evolved from swine in Mexico ${ }^{3}$ and spread to various countries. The first of the human infections were seen in California, and the strain isolated from there. ${ }^{2}$ India was affected with a moderate severity compared to the other countries, although there was an insufficiency of epidemiological data from India during 2009-2010.4 Postpandemic period, India was affected by major outbreaks in 2015 and 2017, with bimodal peaks in winter and summer months of 2017.5 In tropical regions like India, influenza season is seen throughout the year, causing irregular outbreaks.

Influenza is mainly a droplet infection involving the respiratory tract (upper and lower), also transmissible by contact exposure. ${ }^{2}$ Upper respiratory tract like illness, presenting with fever and cough or sore throat without any other diagnosis should rouse suspicion of influenza, especially if the presenting complaint is breathlessness. ${ }^{1}$ Some groups of individuals are at higher risk than others: elderly (65 years and older), young children (less than 2 years), pregnant women, asthmatics, diabetics, history of heart disease or stroke, compromised immune systems secondary to HIV, cancer etc. ${ }^{6}$

A diagnosis is established with Real-Time Reverse Transcription Polymerase Chain Reaction (RT-PCR) performed on a sample of nasopharyngeal or an oropharyngeal swab. ${ }^{2}$ Although the RT-PCR test is being made more readily available, Influenza virus is not routinely tested. Active case-finding with high clinical suspicion is the only way to remain ahead of illness from influenza especially in countries like India. Treatment is recommended with high dose oseltamivir, up to $150 \mathrm{mg}$ twice a day in critically ill patients for a minimum of 5 days. ${ }^{7}$ This need to be supported by symptomatic care and respiratory support as when it is required. It is recommended that the high-risk groups undergo annual vaccination and the group of people in contact with such patients, especially health care workers to receive post exposure prophylaxis with oseltamivir for 7 - 10 days. ${ }^{7}$

We wanted to analyse the rate of positivity in a tertiary care centre amongst the total number of oropharyngeal swabs sent for Influenza virus testing. We also wanted to assess the mortality rate in Influenza positive cases in a tertiary care centre.

\section{METHODS}

This is a cross sectional study carried out between January $1^{\text {st }}$, 2016 to November 22nd 2018 . This was a time bound study and all the cases that were swabbed within the study period were included in this study after appropriate informed consent from the patient's attenders. Inclusion criteria was age of 18 years or older and H1N1 swab being taken. All nonconsenting participants were excluded.

An oropharyngeal swab was taken under aseptic precautions and was transported in a sterile viral transport medium. The number of oropharyngeal swabs sent for influenza virus testing from K.S. Hegde Medical Academy, Deralakatte was reviewed through a retrospective search of official influenza virus reports of Karnataka State along with the reports in Medical Records Department of K.S. Hegde Medical Academy.

\section{Statistical Analysis}

Data was analysed using SPSS software version 25. Results were presented as percentages and pie charts against multiple parameters.

\section{RESULTS}

A total of 127 patients were swabbed to rule out H1N1 in the study period of 1 year and 11 months. Oropharyngeal swabbing was done to rule out H1N1 for various indications.

\section{Why was Influenza Suspected?}

In majority of the cases, 51 out of 127 ( $40.1 \%$ ), the presenting upper respiratory tract symptoms were severe and required Intensive Care Unit (ICU) admission and ventilatory support as Non-Invasive Ventilation (NIV) or mechanical ventilation (Figure 1). In 27 cases (21.3\%) throat swabs were sent due to worsening of initial upper respiratory tract symptoms and in 24 cases $(18.9 \%)$ they were sent as patient was not improving on antibiotics and hence to rule out a viral aetiology. There was history of contact with known H1N1 cases in 2 instances and in 9 cases chest X-Ray was suggestive of Acute Respiratory Distress Syndrome (ARDS) like picture, which warranted further investigation to rule out a viral aetiology.

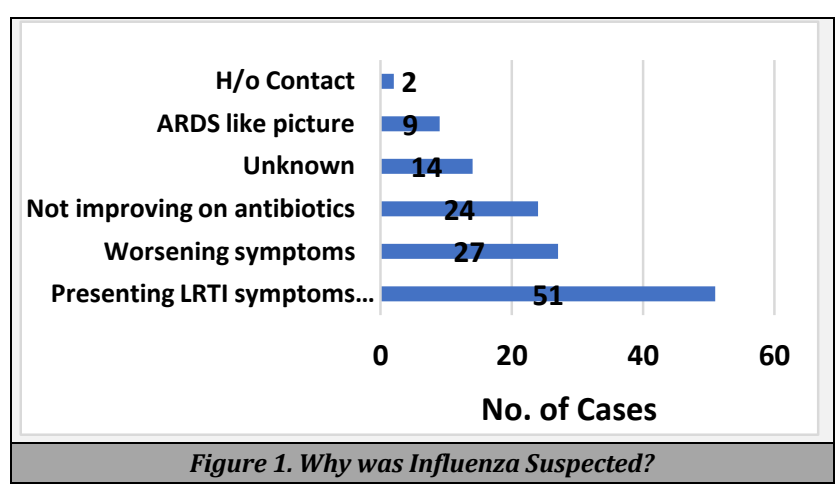

Out of 127 throat swabs that were sent, 24 (18.9\%) were positive for the different strains of the influenza virus (Figure 2). Rest of the 103 cases were negative for influenza. 
18 of the positive 24 cases were found to be positive for the strain of H1N1, 3 were H3N2 positive (seasonal strain) and remaining 3 were positive for influenza $B$ virus. Of the total study population, 18 (14.2\%) cases were positive for H1N1. The remaining 6 cases $(4.7 \%)$ were positive for the other seasonal and less virulent strains of influenza virus.

9 out of 24 cases of influenza succumbed to the disease. All of these $9(7.1 \%)$ cases that died were positive for the H1N1 strain of influenza (Figure 3). Among the total 18 H1N1 positive cases, mortality was seen in $50 \%$ of cases ( 9 out of 18). 4 cases were Discharged Against Medical Advice (DAMA) to a local hospital in view of financial constraints. 11 cases recovered and were discharged in good health.

Overall mortality due to H1N1 virus in the study period in our hospital was found to be $0.6 \%$ (9 out of 1360).

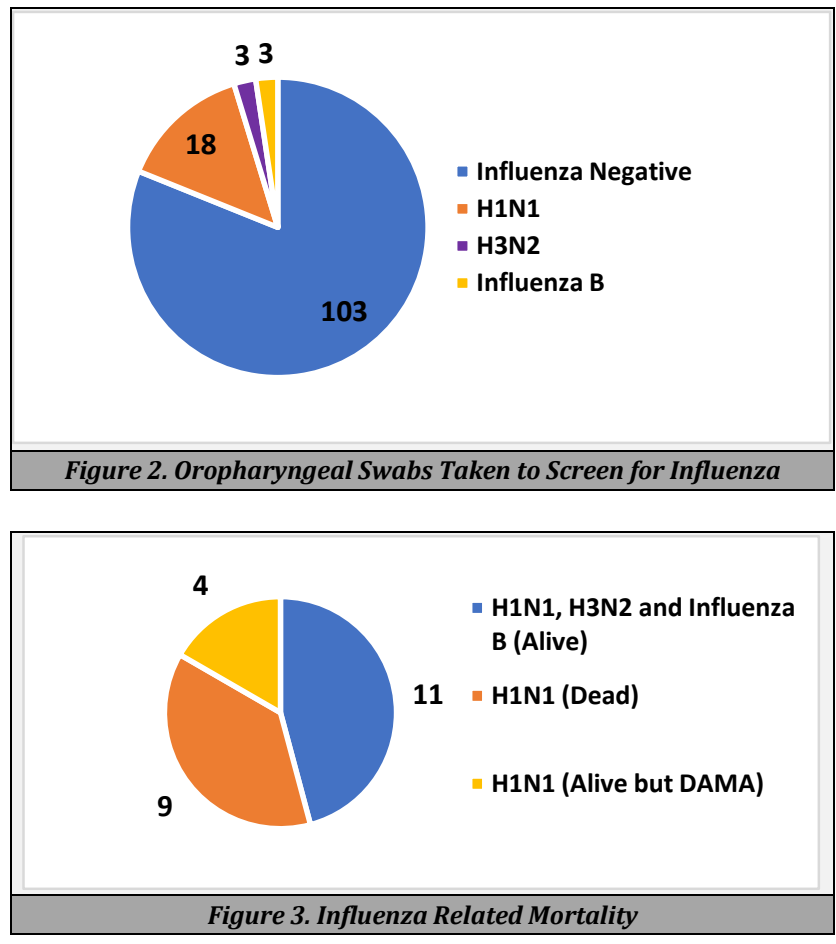

\section{DISCUSSION}

Influenza virus is known to occur in two forms: epidemics and pandemics. ${ }^{8}$ This is due to the phenomenon of antigenic drift, ${ }^{2}$ which causes the strain to alter and evolve to become resistant to existing treatment options. This phenomenon leads to a severe illness and higher mortality in pandemics and affect not only the more susceptible individuals, but all of the population alike. ${ }^{8}$

In 2009 H1N1 pandemic, Pune was the epicentre of the outbreaks. ${ }^{9}$ A study documented a mortality up to one death per 11 confirmed cases that were hospitalized. ${ }^{10}$ The same study also pointed out $8.6 \%$ hospitalization of cases. In 2010, World Health Organization declared post pandemic era. ${ }^{2}$ However the numbers in India due to H1N1 were once again on the rise in 2015 with high mortality recorded in central India. ${ }^{11}$ In 2017, death by H1N1 were eight times the previous years and three times higher than the 2009 pandemic year. ${ }^{5}$ Our small-scale study was conducted in Deralakatte, India in a tertiary care setting between 2016 and 2018 in these times.
A new strain of H1N1 was found responsible for the increase in numbers in India (A / Michigan / 45 / 2015), along with the strain responsible for the pandemic in 2009 (A / California / 7 / 2009). A study in India compared the mortality due to both strains in 2015 and 2018 and concluded a mortality of $16 \%$ and $20 \%$ respectively. ${ }^{12}$ In our study 18 cases were $\mathrm{H} 1 \mathrm{~N} 1$ positive (14.2\%). 9 out of 18 cases died suggesting a $50 \%$ mortality due to $\mathrm{H} 1 \mathrm{~N} 1$ in the study period. Additional $4 \mathrm{H} 1 \mathrm{~N} 1$ cases were discharged against medical advice (DAMA) due to monetary issues. On follow up, all 4 of them succumbed to the disease as well, increasing the mortality exponentially. During this time period, the all-cause mortality in the hospital was $1360,9(0.6 \%)$ of which were due to H1N1. It is interesting to note that all the individuals affected with either the seasonal strain of influenza (H3N2) or influenza B (6 cases) recovered from their infection uneventfully.

\section{CONCLUSIONS}

Respiratory infections are one of the most common causes of death in India, yet there are no statistics available on Influenza related mortality. This is thought to be a consequence of underestimation of Influenza related deaths as routine testing for the virus is not done for patients presenting with respiratory symptoms. H1N1 related mortality is a cause for concern for every healthcare providing institution. Influenza virus should be actively tested in patients with respiratory symptoms. During this COVID-19 related pandemic situation, there is a higher possibility of underdiagnosing H1N1. Lessons learnt from the past should be remembered and Influenza should not be neglected.

Data sharing statement provided by the authors is available with the full text of this article at jemds.com.

Financial or other competing interests: None.

Disclosure forms provided by the authors are available with the full text of this article at jemds.com.

\section{REFERENCES}

[1] World Health Organization. A practical guide for designing and conducting influenza disease burden studies. Geneva: World Health Organization 2009.

[2] Sullivan SJ, Jacobson RM, Dowdle WR, et al. 2009 H1N1 influenza. Mayo Clin Proc 2010;85(1):64-76.

[3] Vijaykrishna D, Poon LL, Zhu HC, et al. Reassortment of pandemic H1N1/2009 influenza a virus in swine. Science 2010;328(5985):1529.

[4] Choudhry A, Singh S, Khare S, et al. Emergence of pandemic 2009 influenza A H1N1, India. Indian J Med Res 2012;135(4):534-7.

[5] Kulkarni SV, Narain JP, Gupta S, et al. Influenza A (H1N1) in India: changing epidemiology and its implications. Natl Med J India 2019;32(2):107-8.

[6] Centers for Disease Control And Prevention. People at high risk for flu complications. USA: US Department of Health and Human Services 2020.

[7] World Health Organization. Pharmacological management of pandemic influenza a (H1N1) 2009 and 
other influenza viruses. Part 1: recommendations. World Health Organization 2010:10-4.

[8] Purohit V, Kudale A, Sundaram N, et al. Public health policy and experience of the $2009 \mathrm{H} 1 \mathrm{~N} 1$ influenza pandemic in Pune, India. Int J Health Policy Manag 2018;7(2):154-66.

[9] Tandale BV, Pawar SD, Gurav YK, et al. Seroepidemiology of pandemic influenza a (H1N1) 2009 virus infections in Pune, India. BMC Infect Dis 2010;10:255.

[10] Mishra AC, Chadha MS, Choudhary ML, et al. Pandemic influenza (H1N1) 2009 is associated with severe disease in India. PLoS One 2010;5(5):e10540.
[11] Barde PV, Sahu M, Shukla MK, et al. The high frequency of non-aspartic acid residues at $\mathrm{HA}_{222}$ in influenza $\mathrm{A}(\mathrm{H} 1 \mathrm{~N} 1)$ 2009 pandemic viruses is associated with mortality during the upsurge of 2015: a molecular and epidemiological study from central India. Epidemiol Infect 2017;145(13):2656-65.

[12] Nawal CL, Agarwal S, Agrawal A, et al. Mortality in H1N1: a comparison of patient attributes in outbreaks due to a/California/7/2009 and A/Michigan/45/2015 strains. J Assoc Physicians India 2019;67(3):38-41. 